\title{
Postprocedural Endophthalmitis or Postprocedural Intraocular Inflammation: A Diagnostic Conundrum
}

\author{
Aisha Al Busaidi Ahmed Al-Hinai \\ Department of Ophthalmology, Sultan Qaboos University Hospital, Al Khoudh, Oman
}

\section{Keywords}

Endophthalmitis · Culture-negative $\cdot$ Intraocular inflammation $\cdot$ Intra-vitreal injection $\cdot A C$ paracentesis

\begin{abstract}
We experienced an atypical endophthalmitis occurring post consecutively performed in-office procedures; an intravitreal injection (IVI) of ranibizumab followed by an anterior chamber (AC) paracentesis performed twice in an eye with neovascular glaucoma (NVG). A 52-year-old diabetic male who was asymptomatic developed signs of endophthalmitis and decreased vision without pain in his left eye a few days post-IVI and AC paracentesis. The condition worsened after an initial vitreous tap and injection of antibiotics. Cultures of vitreous and aqueous samples were negative. Complete resolution occurred after a pars plana vitrectomy with IVI of antibiotics and steroid with removal of a dense "yellowish-brown" fibrinous plaque. The absence of pain, presence of a peculiar colored fibrin, mild-to-moderate vitritis without retinitis, negative cultures, and complete recovery despite the fulminant presentation; favor a diagnosis of inflammation over infection. We hypothesize that a micro-leak from a 26-gauge AC tap tract might have served as an entry port for $5 \%$ povidone-iodine from the ocular surface thus inciting inflammation. However, an exuberant inflammatory response that can be typically seen in NVG eyes after intraocular procedures cannot be excluded. Various causes of inflammation post-procedures, both toxic and nontoxic should be considered in atypical culture-negative fulminant endophthalmitis cases with good outcome posttreatment. Any minor ocular procedure may carry a risk of such complication. Patient counseling and care must be exercised in performing these procedures.
\end{abstract}


Al Busaidi and Al-Hinai: A Case of Postprocedural Endophthalmitis or Intraocular Inflammation

\section{Introduction}

Post-procedural endophthalmitis is a rare but devastating event. The incidence of endophthalmitis from intravitreal injections (IVIs) ranges from 0.03 to $0.87 \%$ per injection [1]. Anterior chamber (AC) paracentesis is not free of complications. Helbig et al. [2] reported a case of bacterial endophthalmitis after an AC paracentesis performed in an operating room. Both infectious and noninfectious endophthalmitis have been reported as complications of IVIs [3-5]. Many noninfectious entities can mimic infectious endophthalmitis, such as sterile endophthalmitis, a variant of toxic anterior segment syndrome involving the posterior segment which has been recently coined as toxic posterior segment syndrome (TPSS) or an inflammatory process related to the underlying disease itself. It is sometimes challenging to differentiate these conditions due to overlapping features. We describe here a unique fulminant but culture-negative endophthalmitis occurring after 2 types of procedures; an IVI of anti-VEGF and concurrent AC paracentesis, followed by a second paracentesis the next day.

\section{Case Report}

A 52-year-old known diabetic male was diagnosed with a central retinal vein occlusion and secondary macular edema with neovascular glaucoma (NVG) in the left eye (OS). His bestcorrected visual acuity at presentation was counting fingers (CFs) OS. An IVI of ranibizumab $0.5 \mathrm{mg}$ with a concomitant $\mathrm{AC}$ paracentesis was performed as an in-office procedure following an aseptic technique and using a speculum and face mask. Post-procedure, topical ofloxacin, and aqueous suppressants were prescribed. He was scheduled for definitive treatment with pan-retinal photocoagulation in the following week.

A day later, the patient returned to the emergency department with severe eye pain OS. He was found to have an IOP of $60 \mathrm{~mm} \mathrm{Hg}$ with a $1 \mathrm{~mm}$ hyphema and diffuse corneal edema. The ocular pain was attributed to the acute rise in IOP due to the hyphema from the underlying iris neovascularization. An emergent $\mathrm{AC}$ paracentesis was performed at the slit lamp to relieve the ocular pressure and pain. It was carried out according to a standard technique using topical anesthetic followed by $5 \%$ povidone-iodine antiseptic solution into the conjunctival sac. Using an eye speculum and a sterile 26-gauge fixed-needle tuberculin syringe, aqueous fluid was drained. Immediately post-procedure, the IOP was reduced to $2 \mathrm{~mm} \mathrm{Hg}$ and copious amounts of povidone-iodine and anti-glaucoma drops were re-instilled. Half an hour later, his IOP had normalized and a small area of scanty leakage of fluid was noted from the entry site of the needle, but the AC remained deep. He was sent home on topical antibiotic, maximal anti-glaucoma medications and told to follow-up in 2 days.

On reassessment 2 days later, he was incidentally found to have vision reduced from a baseline of CF to hand motion, associated with conjunctival injection, a $2 \mathrm{~mm}$ hypopyon overlying a layer of hyphema, a mild fibrinous reaction in the AC, and a hazy cornea without keratic precipitates on the corneal endothelium. He was asymptomatic and denied having any ocular pain. There was limited view of the retina and a B-scan revealed mild-to-moderate vitritis (shown in Fig. 1). A diagnosis of acute post-procedural infectious endophthalmitis was suspected, and the patient underwent an immediate tap of vitreous with injection of intravitreal antibiotics. A volume of $0.2 \mathrm{~mL}$ of vitreous was aspirated through the pars plana for gram stain, cultures, and sensitivities. The patient empirically received intravitreal vancomycin $(1 \mathrm{mg} / 0.1 \mathrm{~mL})$ and ceftazidime $(2.25 \mathrm{mg} / 0.1 \mathrm{~mL})$. Topical fortified cefuroxime and gentamicin alternating every half an hour together with atropine drops were started. An oral ciprofloxacin loading dose of $1 \mathrm{~g}$ was given and maintained on $750 \mathrm{mg}$ twice daily as well as oral prednisolone $30 \mathrm{mg}$ once daily.

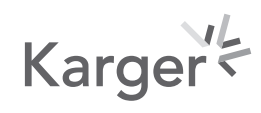


Al Busaidi and Al-Hinai: A Case of Postprocedural Endophthalmitis or Intraocular Inflammation

Fig. 1. B-scan ultrasonography of the OS. Demonstrates mild-to-moderate opacities in the vitreous cavity indicative of vitritis. OS, left eye.

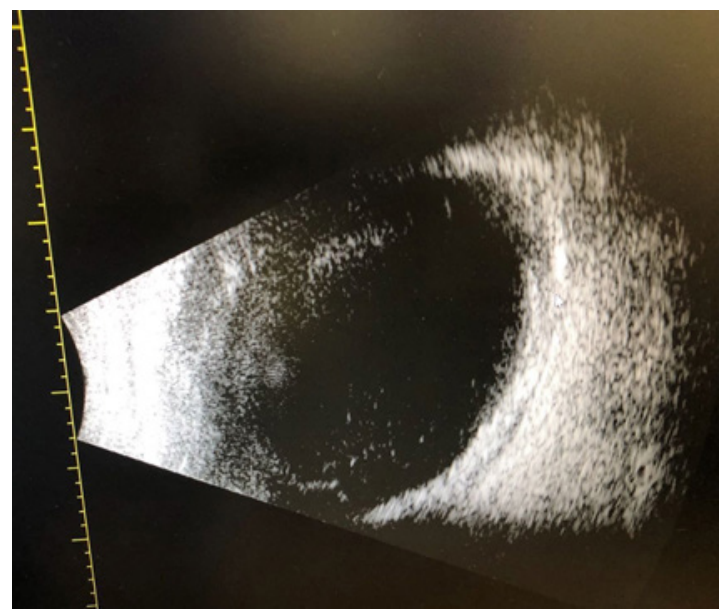

Hours later, there was pronounced AC fibrinous reaction, obscuring the iris and lens. He was then started on hourly prednisolone acetate $1 \%$ drops. On the following day, he remained asymptomatic, but his vision dropped further to light perception. On slit-lamp exam, there was worsening corneal edema and a densely organized fibrinous membrane seen in the AC. Initial smears of vitreous sample showed no pus cells or organisms, and so an aqueous sample $(0.1 \mathrm{~mL})$ at this point was additionally withdrawn for microbiological analysis. In view of the worsening picture, an emergency 23-gauge pars plana vitrectomy (PPV) with AC washout, endo-laser pan-retinal photocoagulation was performed and IVI of ceftazidime, vancomycin, and dexamethasone were administered. Intraoperatively, the membranous plaque was peeled off and found to be of a peculiar dense "yellow-brown" in color (shown in Fig. 2). The vitreous lacked dense opacities or membranes but rather a moderate amount of white particulate matter floating within. There was no retinitis or periphlebitis. A vitreous biopsy was obtained during vitrectomy and sent for microbiological analysis. Postoperatively, his vision improved back to baseline (i.e., CF). Fibrinous remnants in the AC gradually settled as topical and oral prednisolone were tapered. All vitreous and aqueous samples resulted negative on microscopy and culture. By the tenth-day post op, the AC and cornea were completely cleared with no sequelae.

\section{Discussion}

There is no reliable way that can clearly distinguish post-procedural infectious endophthalmitis from noninfectious inflammation [6]. Some strong predictors of a possible infectious process in our case were the presence of hypopyon and fibrin in the AC as well as sudden visual loss. One study reported hypopyon as a presenting feature of infectious endophthalmitis in 78\% of patients [7]. The asymptomatic nature of the presentation and absence of pain did not typically favor an infectious etiology. However, pain is highly variable and subjective, and we can reason that it may be absent in diabetic patients who are neuropathic. Our suspicion of infection was also not supported by vitreous or aqueous cultures. But it is known that negative cultures do not exclude the diagnosis; as up to $30 \%$ of endophthalmitis cases are culture negative. This may be a result of false negatives including inadequate, inappropriate sampling, presence of fastidious organisms, or a low pathogen load that may be undetectable on culture. Other differential diagnosis includes a true "sterile" endophthalmitis, a 

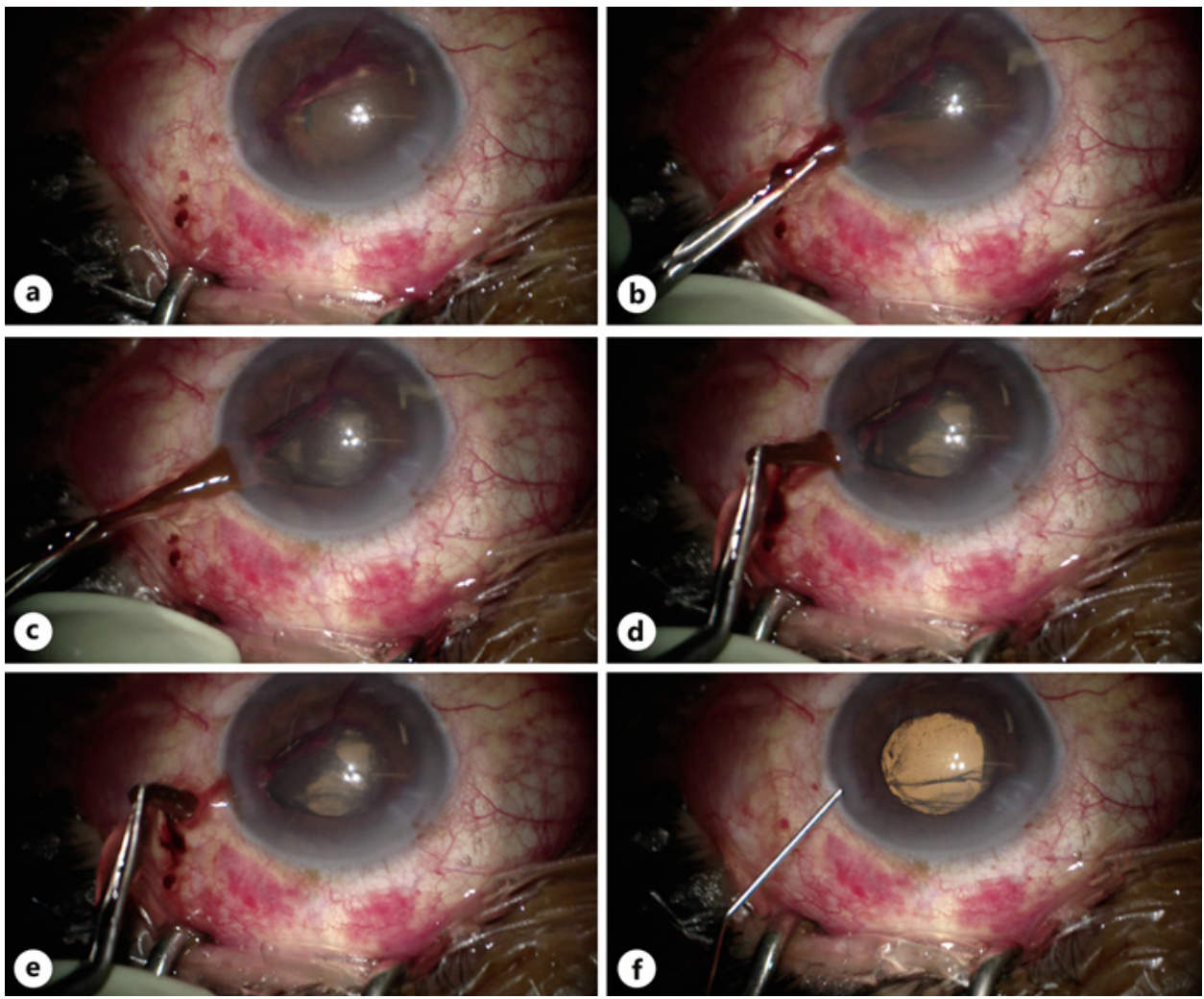

Fig. 2. Intraoperative anterior segment photographs of the OS. a A small "candy-striped hyphema" is seen inferiorly with a dark "yellowish-brown" densely organized fibrin spanning the AC obscuring the iris, lens, and pupil details. b-e The fibrin plaque, which is indistinguishable from the patient's brown iris color, is seen peeled off the surface of the lens and iris using micro-forceps. $\mathbf{f}$ AC washout post-membrane peeling reveals a clear AC, round pupil with a good fundus glow seen through it. AC, anterior chamber; OS, left eye.

variant of toxic anterior segment syndrome, or masquerade syndromes. In these instances, the use of alternate investigational modalities such as fast real-time PCR or next-generation sequencing may have helped increase the diagnostic yield for microbes $[8,9]$.

The specific inciting event that led to this iatrogenic complication cannot be proven with certainty as the ocular inflammation occurred within 3 days of an uneventful IVI of ranibizumab and within 2 days after a repeat AC paracentesis. In our case, all procedures were performed adhering to latest preferred practice patterns. We retrospectively assessed risk factors for the development of endophthalmitis. Preoperatively, the patient was diabetic, which is a known factor for development of acute-onset postoperative endophthalmitis. Intraoperatively, there was povidone-iodine antisepsis used during both procedures, which is the only proven agent known to reduce the risk of endophthalmitis [10]. When administering IVI, aside from wearing a face mask, which was used in our case, no other modifiable risk factors were found to be significant in the literature [11]. Postoperatively, the patient was prescribed topical antibiotic prophylaxis to be used postinjection, but no study has shown benefit of this in preventing postinjection endophthalmitis [12].

The underlying etiology of the condition was ambiguous. The onset of symptoms in infectious endophthalmitis typically correlates with the virulence of the causative organism. In our case, the acute fulminant picture (i.e., occurring within 4 days of the presumed inciting event) and the rapid progression, led us to initially believe the condition was due to a virulent or 
Al Busaidi and Al-Hinai: A Case of Postprocedural Endophthalmitis or Intraocular Inflammation

atypical microorganism. However, both vitreal (initial and repeat) and aqueous cultures failed to identify an offending microorganism to corroborate our suspicions. In addition, the complete resolution and good clinical outcome after subsequent vitrectomy did not reflect an outcome that is typically seen with virulent organisms. The atypical features of lack of pain, absence of retinitis, low-to-moderate level of vitritis seen on echography and intraoperatively during PPV, together with culture negativity, made us entertain alternate diagnosis, one of which is "sterile" endophthalmitis postinjection. Potential causes of this are immune reaction to the drug itself, its preservatives, to breakdown products in the injected material or endotoxins or to an unknown contaminant [13]. However, by definition, this condition resolves without the need of intravitreal antibiotics and/or vitreoretinal surgery and, therefore, cannot be entertained with certainty in our case since interventions were performed and were effective in resolving the condition.

Based on the unique features in our case, what can be suggested is a diagnosis of TPSS (toxic endophthalmitis). This is similar to what was reported by LoBue and LoBue [14] after uneventful cataract surgery. The condition mimicked fulminant culture-negative bacterial endophthalmitis but had a good visual outcome posttreatment. Their explanation was that a toxic stimulus entered the $\mathrm{AC}$ and made its way posteriorly developing an intense inflammatory reaction that also involved the posterior segment. A clue strongly in favor of this in our patient was the "yellow-brown" fibrinous plaque which closely resembled the color of povidone-iodine. This peculiar discoloration was clearly not attributed to the presence of erythrocytes in the AC as the hyphema that was also present had insufficient time to be altered or its cells degenerated. We hypothesize that povidone-iodine on the ocular surface operculated through the 26-gauge needle tract either through the small wound leak or sucked in by negative pressure due to the pressure difference between the AC and surrounding atmospheric pressure after the precipitous drop in IOP causing an influx of solution into the eye especially with the copious instillation of eye drops immediately post-procedure. Although variable-sized needles are typically used for AC paracentesis (i.e., ranging from 25- to 30-gauge needles), no study has established major differences in their complication rates, but the use of smaller gauge needles such as 30-gauge compared to a larger 26-gauge may theoretically help minimize such complications. The 5\% antiseptic solution, proven useful on the ocular surface, could have incited inflammation once in the AC [15]. Shimada et al.[16] have concluded that safe intravitreal povidone-iodine concentration was estimated to be no more than $0.027 \%$.

Finally, we cannot exclude the possibility of an ocular inflammatory response seen in response to a generalized ischemic state in NVG eyes and one that is exacerbated by intraocular procedures. Eyes with NVG, formerly known as "congestive glaucoma," are inflamed eyes, typically known for exhibiting conjunctival congestion and variable degrees of iritis and vitritis. Although the presence of a hypopyon is a rarity in typical cases of NVG, it is conceivable that an intense inflammatory state producing an unusually large number of cells in the AC can produce a sterile hypopyon with sedimentation of these cells. Similar to the rare occurrence of hypopyons in acute angle-closure glaucomas, previous reports have documented their occurrence 2-3 days post-resolution of acute angle closure $[17,18]$. Schartz et al. [17] have suggested a postischemic vascular leakage of leukocytes into the AC occurring from reperfusion of the ischemic damaged iris as the IOP is lowered, thereby resulting in a rapid exudation of white cells in the AC. Our patient's hypopyon had similarly been seen 2 days after IOP lowering with an AC tap. With steroid treatment, the fibrinous reaction further consolidated covering the visual axis, thereby leading to a paradoxical reduction in vision despite there being actually an improvement in inflammation. The fibrinous plaque, intermixed with RBCs of the hyphema can alternatively explain the discolored yellow-brown fibrin in the AC.

The clearing of the condition with PPV may be the case in any of the possible etiologies as it debulks the vitreous from organisms, toxins or inflammatory cells, and debris. Despite

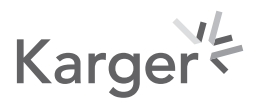


Al Busaidi and Al-Hinai: A Case of Postprocedural Endophthalmitis or Intraocular Inflammation

this effective treatment, there was no permanent damage or deleterious visual sequelae noted unlike what would be expected with atypical virulent infections.

\section{Conclusion}

We present a diagnostically challenging endophthalmitis occurring post-IVI and AC paracentesis. Our hypothesis favors a diagnosis of inflammation over infection. The most likely inflammatory diagnosis is toxic endophthalmitis (TPSS) secondary to povidone-iodine contamination post AC tap, although we cannot exclude an exuberant inflammatory response exhibited by NVG eyes in response to intraocular procedures. However, in such unclear cases, it is best to promptly treat empirically as an infectious endophthalmitis.

\section{Acknowledgement}

The authors thank Dr. Nawal Al Fadhil, Sultan Qaboos University Hospital, Oman for her assistance in delivering ophthalmic care to the patient.

\section{Statement of Ethics}

The patient described in this case report provided informed consent for its publication and any accompanying images. This study does not include any information which may reveal the patient's identity. The procedures conformed to the tenets of the World Medical Association's Declaration of Helsinki.

\section{Conflict of Interest Statement}

The authors have no conflicts of interest to declare.

\section{Funding Sources}

No funding was received for this study.

\section{Author Contributions}

Dr. Al Busaidi wrote the manuscript and contributed to patient management. Dr. Al-Hinai reviewed the manuscript and treated the patient. All the authors read, reviewed, edited, and approved the manuscript. They equally participated in the analysis of the case.

\section{Karger'}


Al Busaidi and Al-Hinai: A Case of Postprocedural Endophthalmitis or Intraocular Inflammation

\section{References}

1 Levison AL, Mendes TS, Bhisitkul R. Postprocedural endophthalmitis: a review. Expert Rev Ophthalmol. 2013; 8(1):45-62.

2 Helbig H, Noske W, Kleineidam M, Kellner U, Foerster MH. Bacterial endophthalmitis after anterior chamber paracentesis. Br J Ophthalmol. 1995;79(9):866.

3 Moshfeghi AA, Rosenfeld PJ, Flynn HW Jr, Schwartz SG, Davis JL, Murray TG, et al. Endophthalmitis after intravitreal vascular [corrected] endothelial growth factor antagonists: a six-year experience at a university referral center. Retina. 2011;31(4):662-8.

4 Day S, Acquah K, Mruthyunjaya P, Grossman DS, Lee PP, Sloan FA. Ocular complications after anti-vascular endothelial growth factor therapy in Medicare patients with age-related macular degeneration. Am J Ophthalmol. 2011;152(2):266-72.

5 Chong DY, Anand R, Williams PD, Qureshi JA, Callanan DG. Characterization of sterile intraocular inflammatory responses after intravitreal bevacizumab injection. Retina. 2010;30(9):1432-40.

6 Schwartz SG, Flynn HW Jr, Emerson GG, Choudhry N, Ferrone PJ, Goldberg RA, et al. Distinguishing between infectious endophthalmitis and noninfectious inflammation following intravitreal anti-VEGF injection. J Vitreoretin Dis. 2019;3(1):42-4.

7 Fintak DR, Shah GK, Blinder KJ, Regillo CD, Pollack J, Heier JS, et al. Incidence of endophthalmitis related to intravitreal injection of bevacizumab and ranibizumab. Retina. 2008;28(10):1395-9.

8 Chakrabarti M, John SR, Chakrabarti A. Culture negative (sterile) postoperative endophthalmitis. Kerala J Ophthalmol. 2009;21(2):190-3.

9 Deshmukh D, Joseph J, Chakrabarti M, Sharma S, Jayasudha R, Sama KC, et al. New insights into culture negative endophthalmitis by unbiased next generation sequencing. Sci Rep. 2019;9(1):844.

10 Ta CN. Minimizing the risk of endophthalmitis following intravitreous injections. Retina. 2004;24(5):699705.

11 Shah CP, Garg SJ, Vander JF, Brown GC, Kaiser RS, Haller JA. Outcomes and risk factors associated with endophthalmitis after intravitreal injection of anti-vascular endothelial growth factor agents. Ophthalmology. 2011;118(10):2028-34.

12 Cheung CS, Wong AW, Lui A, Kertes PJ, Devenyi RG, Lam WC. Incidence of endophthalmitis and use of antibiotic prophylaxis after intravitreal injections. Ophthalmology. 2012;119(8):1609-14.

13 Orozco-Hernández A, Ortega-Larrocea X, Sánchez-Bermúdez G, García-Aguirre G, Cantón VM, Velez-Montoya R. Acute sterile endophthalmitis following intravitreal bevacizumab: case series. Clin Ophthalmol. 2014;8: 1793-9.

14 LoBue S, LoBue TD. Fulminant culture negative endophthalmitis as a new entity, toxic posterior segment syndrome? Adv Ophthalmol Vis Syst. 2016;4(6):171-5.

15 Alp BN, Elibol O, Sargon MF, Aslan OS, Yanyali A, Karabas L, et al. The effect of povidone iodine on the corneal endothelium. Cornea. 2000;19(4):546-50.

16 Shimada H, Arai S, Nakashizuka H, Hattori T, Yuzawa M. Reduced anterior chamber contamination by frequent surface irrigation with diluted iodine solutions during cataract surgery. Acta Ophthalmol. 2017;95(5):e373-8.

17 Schartz SD, Borchert M, Oberman A. Hypopyon keratouveitis in acute angle-closure glaucoma. Am J Ophthalmol. 1987;104(4):430-1.

18 Friedman AH, Bloch R, Henkind P. Hypopyon and iris necrosis in angle-closure glaucoma. Report of two cases. Br J Ophthalmol. 1972;56(8):632-5. 